\title{
Obesidad en adolescentes y criterios para el desarrollo de síndrome metabólico
}

\section{Adolescent obesity and related metabolic syndrome development criteria}

\section{Obesidade em adolescentes e critérios para o desenvolvimento de síndrome metabólica}

\author{
H. Ávila-Alpirez ${ }^{\mathrm{a}}$, G. Gutiérrez-Sánchez ${ }^{\mathrm{a}}$, J. Guerra-Ordoñez ${ }^{\mathrm{a} *}$, J. Ruíz-Cerino ${ }^{\mathrm{a}}$, M. Martínez-Aguilar ${ }^{\mathrm{a}}$ \\ ${ }^{a}$ Unidad Académica Multidisciplinaria Matamoros, Universidad Autónoma de Tamaulipas, H. Matamoros, Ta- \\ maulipas, México
}

Recibido: 12 febrero 2018

Aceptado: 31 julio 2018

\section{Resumen}

Objetivo: El objetivo de este trabajo es determinar los criterios para síndrome metabólico (SM) de mayor influencia en adolescentes con obesidad.

Método: El estudio fue de tipo descriptivo y transversal, la muestra la conformaron 122 adolescentes de nivel preparatoria, seleccionados por un muestreo aleatorio simple. Se realizó somatometría (peso, talla y circunferencia de cintura), así como medición de la presión arterial y toma de muestra sanguínea.

Resultados: Predominó la obesidad grado I (68\%), el principal criterio para el diagnóstico de síndrome metabólico fue la obesidad abdominal en el $98.4 \%$ de los participantes; el 25.4\% de estos presentaron tres o más criterios alterados por lo que se consideran con SM, además se encontró asociación entre los niveles de glicemia con la obesidad $(\mathrm{p}<0.01)$.

Conclusiones: Los hallazgos de esta investigación mostraron elevada prevalencia de SM en los participantes. La obesidad abdominal es un claro signo de problemas relacionados con el peso y se presenta muy frecuentemente en la población joven con obesidad. La adolescencia es una etapa temprana para educar a las personas y fomentar una vida más sana que permita mantenerlos lejos del desarrollo de enfermedades crónico degenerativas.

Palabras clave: Síndrome metabólico; obesidad; adolescentes; México.

\section{Abstract}

Objective: To identify the main metabolic syndrome (MS) criteria in adolescents with obesity.

\footnotetext{
* Autor de correspondencia. Correo electrónico: jesus.guerra@docentes.uat.edu.mx http: //dx.doi.org/10.22201/eneo.23958421e.2018.4.534 
Methods: This is a descriptive and transversal study. The sample was constituted by 122 high school level adolescents selected by simple aleatory sampling. Weight, size, waist circumference, artery pressure, and a blood sample were taken.

Results: Obesity of degree I was prevalent (68\%). The main criterion for the diagnosis of metabolic syndrome was the abdominal obesity, which was found in $98.4 \%$ of the sample. $25.4 \%$ of these participants also showed 3 or more metabolic syndrome related criteria. An association between the levels of glycaemia and obesity was also found $(\mathrm{p}<0.01)$.

Conclusions: The findings revealed a high metabolic syndrome prevalence among the participants. Having abdominal obesity is a clear sign of problems related to weight which, frequently, is present among young populations with obesity. The adolescence is an early stage when people can be educated to live a healthy life and prevent chronic-degenerative illnesses.

Keywords: Metabolic syndrome; obesity; adolescents; Mexico.

\section{Resumo}

Objetivo: O objetivo deste trabalho é determinar os critérios para síndrome metabólica (SM) de maior influência em adolescentes com obesidade.

Método: El estudo foi de tipo descritivo e transversal, a amostra a conformaram 122 adolescentes de ensino médio, selecionados por uma amostra aleatória simples. Realizou-se somatometria (peso, tamanho e circunferência de cinto), assim como medição da pressão arterial e tomada de amostra sanguínea.

Resultados: Predominou a obesidade grau I (68\%), o principal critério para o diagnóstico de síndrome metabólica foi a obesidade abdominal no $98.4 \%$ dos participantes; o $25.4 \%$ destes apresentaram três ou mais critérios alterados pelo que se consideram com SM, além disso encontrou-se associação entre os níveis de glicemia com a obesidade $(\mathrm{p}<0.01)$.

Conclusões: Os achados desta pesquisa mostraram elevada prevalência de SM nos participantes. A obesidade abdominal é um claro signo de problemas relacionados com o peso e se apresenta muito frequentemente na população jovem com obesidade. A adolescência é uma fase inicial para educar às personas e fomentar uma vida mais saudável que permita mantê-los longe do desenvolvimento de doenças crónico degenerativas.

Palavras chave: Síndrome metabólica; obesidade; adolescentes; México.

\section{Introducción}

El sobrepeso y la obesidad se definen como una acumulación anormal o excesiva de grasa que puede ser perjudicial para la salud ${ }^{1}$. El incremento explosivo de la obesidad en adolescentes en todo el mundo en las últimas décadas, ha traído consigo el desarrollo de complicaciones a edades cada vez más tempranas², se ha observado que la ingesta alimenticia perdió calidad nutrimental durante las últimas dos décadas, dado que aumentó el consumo de cereales, aceites, grasas y bebidas gaseosas, entre otros alimentos ricos en carbohidratos ${ }^{3}$.

De acuerdo a los resultados de la Encuesta Nacional de Salud y Nutrición de Medio Camino (ENSANUT 2016), elaborada ante el acelerado incremento en el número de niños, adolescentes y adultos, tanto mujeres como hombres, con sobrepeso y obesidad, y de la aparición de enfermedades relacionadas con la nutrición como diabetes, anemia e hipertensión; se reportó que en adolescentes de entre 12 y 19 años, 4 de cada 10 presentan sobrepeso u obesidad (36.3\%), dato superior a la prevalencia en $2012(34.9 \%)^{4}$.

Así mismo, el incremento en la prevalencia de sobrepeso en adolescentes del sexo femenino fue 2.7 puntos porcentuales, pasó de $23.7 \%$ en 2012 a 26.4\% en 2016. En cambio, la prevalencia de obesidad se mantuvo similar a la de $2012(12.8 \%$ vs $12.1 \%)$. La prevalencia combinada de sobrepeso y obesidad en mujeres adolescentes fue de $39.2 \%$. En adolescentes del sexo masculino se presentó una reducción de $34.1 \%$ a $33.5 \%$ en prevalencia combinada ${ }^{4}$. 
Los problemas de sobrepeso y obesidad pueden producir diversas complicaciones; hace un tiempo se pensaba que los jóvenes con obesidad no desarrollaban problemas cardiovasculares hasta que llegaban a la edad adulta, sin embargo, se ha documentado, que los menores con sobrepeso y obesidad pueden tener complicaciones cardiovasculares a corto y largo plazo 5 . Las personas con obesidad tiene hasta tres veces mayor riesgo de desarrollar diabetes, dado que entre mayor es el Índice de masa corporal (IMC), mayor es la cantidad de grasa corporal, esta grasa proporciona energía al músculo en detrimento de la glucosa, lo que provoca un aumento de la misma. Por consecuente, el páncreas produce mayor cantidad de insulina hasta llegar a un estancamiento, y tiende a ser incapaz de secretar la suficiente cantidad de esta, por lo que la glicemia ya no es regulada de forma exitosa, lo que conlleva a la hiperglicemia y posteriormente al desarrollo de diabetes ${ }^{6}$.

Además, de las complicaciones antes mencionadas, la presencia de sobrepeso es el factor de riesgo más importante para el desarrollo de síndrome metabólico $(\mathrm{SM})^{7}$. En adolescentes, la presencia de sobrepeso (SP) y obesidad (OB), se asocia positivamente con mayor adiposidad, tanto periférica como central, ésta última asociada al SM, por lo que éstas entidades clínicas aparecen como el factor de riesgo más importante para el síndrome ${ }^{8}$. En la actualidad el SM es considerado como una importante forma de evaluar riesgo cardiovascular y diabetes; y está claro que no se trata de una simple definición, sino de un conjunto de anormalidades relacionadas, que por una combinación de factores genéticos y de riesgo, como alteración de estilos de vida (la sobrealimentación y la inactividad o disminución de actividad física), favorecen el desarrollo de las alteraciones fisiológicas asociadas al síndrome ${ }^{9}$.

El SM incluye la obesidad, niveles altos de triglicéridos, bajos niveles de colesterol de lipoproteína de alta densidad (HDL), hipertensión y resistencia a la insulina, ha ganado importancia debido a su asociación con el desarrollo subsecuente de enfermedades cardiovasculares (ECV) y diabetes mellitus tipo $2(\mathrm{DM} 2)^{10}$. Esta fisiopatología está basada principalmente en la resistencia a insulina, como origen del conjunto de anormalidades que conforman el síndrome, intervienen también factores genéticos y ambientales, que van a influir sobre el tejido adiposo y la inmunidad innata ${ }^{11}$.

En revisiones de literatura detalladas, la prevalencia de este síndrome va de 4.2 a $15.4 \%$ con base en los criterios del Programa Nacional de Educación sobre el Colesterol y el Panel III de Tratamiento del Adulto (National Cholesterol Education Program Adult Treatment Panel III), [NCEP-ATP III] y de 4.5 a $38.7 \%$ según los criterios de la Organización Mundial de la Salud (OMS); el componente con mayor frecuencia encontrado generalmente es la hipertrigliceridemia ${ }^{10}$.

De acuerdo con los criterios NCEP-ATP III, una persona debe presentar al menos 3 de los 5 componentes del fenotipo de $\left.\operatorname{Cook}^{12}: 1\right)$ Obesidad abdominal: perímetro de cintura mayor o igual al P90 del referente The Third National Health and Nutrition Examination Survey (NHANES III), por edad y sexo para la población de 2 a 20 años; 2) Hipertensión arterial: presión sistólica o diastólica igual o superior a P90 (de acuerdo a la edad / talla y sexo) establecida por el National High Blood Pressure Education Program Working Group on Hypertension Control in Children and Adolescents de Estados Unidos; 3) Colesterol-HDL bajo en suero: valores de $\leq 40 \mathrm{mg} / \mathrm{dL}$, según el National Cholesterol Education Program (NCEP), para concentración de lípidos en adolescentes; 4) Hipertrigliceridemia: valores en ayunas de $\geq 110 \mathrm{mg}$ / dL, de acuerdo al NCEP para concentración de lípidos en adolescentes; y 5) Hiperglucemia: alteración de la glucemia en ayunas de $\geq 100 \mathrm{mg} / \mathrm{dL}$, de conformidad con los criterios de la Asociación Americana de Diabetes ${ }^{10}$.

En México se han realizado estudios que analizan algunos de los componentes del SM en los adolescentes, sin embargo, son escasos los que valoran el SM en conjunto ${ }^{13-15}$. En las instituciones, es común que distintos servicios realicen labores asiladas, en asistencia e investigación; por lo tanto, no existen lineamientos, programas o abordajes multidisciplinarios coordinados dentro de las instituciones, ni entre ellas. También los esfuerzos que se realizan por los institutos nacionales de salud, hospitales federales de referencia y hospitales de alta especialidad para el desarrollo de investigación básica y clínica de la 
obesidad, son numerosos; pero la difusión de los resultados y la comunicación entre los investigadores aún no es suficiente ${ }^{1}$.

Esto indica que existe la necesidad de realizar investigación por parte de enfermería, en relación a este tema de gran relevancia, tanto para su contribución al cuerpo de conocimientos de la disciplina, así como para contar con un precedente que permita detectar a tiempo criterios para el desarrollo de SM y crear nuevos modelos de atención de enfermería que ayuden a mejorar la práctica profesional. Por lo tanto, realizar una investigación con datos actuales sobre una población en riesgo como los adolescentes con problemas de obesidad resulta indispensable. Con base en lo anterior el objetivo de este trabajo fue determinar los criterios para síndrome metabólico de mayor influencia en adolescentes con obesidad.

\section{Métodos}

El diseño fue descriptivo ${ }^{16}$, y de corte transversal, la población de estudio estuvo conformada por 178 estudiantes con obesidad, de 15 a 19 años de edad, residentes de la ciudad de H. Matamoros, Tamaulipas, estudiantes de nivel medio superior $\left(1^{\circ}, 2^{\circ}\right.$ y $3^{\circ}$ grado de preparatoria), del sector público. El muestreo fue de tipo probabilístico aleatorio simple, el tamaño de la muestra se calculó para proporciones donde se consideró un nivel de confianza del 95\% y un margen de error del 5\%, con una variabilidad estándar de $50 \%$. Por lo que se obtuvo un total de 122 participantes.

Para este trabajo se consideraron como criterios de inclusión participantes inscritos en el Colegio de Bachilleres del Estado de Tamaulipas (COBAT) Plantel 02 de la Heroica Ciudad de Matamoros, de ambos sexos y que presentaran obesidad (Índice de masa corporal mayor al percentil de 95 según edad y género, según criterios de corte del Centers for Disease Control and Prevention (CDC). Así mismo, se excluyeron aquellos participantes con diagnóstico previo de DM2, hipertensión arterial y problemas de coagulación; así como aquellos que estuvieran bajo régimen de medicamentos antidiabéticos, esteroides anabólicos, andrógenos o glucocorticoides. Además, dado que se tomaron muestras de sangre, se excluyeron a aquellos participantes que no contaran con 10 horas de ayuno así como a adolescentes embarazadas.

Se solicitó al plantel la autorización del director para realizar el presente estudio, se obtuvieron las listas y a continuación se agendaron fechas de las actividades para la recolección de los datos. Previo al día de inicio de la recolección, se capacitó a los colaboradores que realizaron las mediciones para que estas estuvieran estandarizadas, y mantener una uniformidad en el proceso de levantamiento de datos. Para los datos somatométricos y la aplicación de la cédula de datos personales, se solicitó la presencia de los estudiantes de acuerdo a la agenda y se presentaron en grupos de 10 personas. Una vez dentro se explicaba en qué consistía el estudio, así como el objetivo y el procedimiento, además de las dudas que surgían, se asignaba un folio (letra y número).

Posteriormente, se procedía a realizar la somatometría (peso, estatura y circunferencia abdominal) con equipo de la marca SECA (báscula, estadiómetro y cinta de fibra de vidrio).

Se calculó el IMC mediante el peso $(\mathrm{Kg}) /$ talla $(\mathrm{m})^{2}$, se clasificaron a los adolescentes por medio de las tablas de percentil (P) del Centro de Salud y Enfermedades de Estados Unidos (CDC/ NCHS), según su edad y sexo como: desnutridos, debajo del P5; peso normal entre P5 y P85; riesgo de sobrepeso, entre P85 y P95; sobrepeso u obesidad igual o mayor a P95. Una vez identificados los sujetos con obesidad se procedió a calcular la muestra, a los seleccionados se les realizó la invitación para la toma de la muestra sanguínea y presión arterial, para esto se les entregó un consentimiento informado el cual debía ser firmado por ambos padres o tutores legales.

Una vez firmados los consentimientos, se agendaron a los participantes y se les indicaba que debían tener 10 horas de ayuno. Si el paciente estaba apto para la toma se procedía a aplicarle la cédula de datos personales, la toma de presión arterial y la toma de la muestra sanguínea, misma que fue extraída por químicos fármaco-biólogos. Para la determinación de glucosa se usó el método de glucosa-oxidasa; el colesterol HDL y los triglicéridos se midieron con un kit enzimático. Las muestras fueron procesadas 
en un laboratorio de análisis clínico local y de prestigio. Después de la toma de la muestra, se dio a los jóvenes un jugo de frutas, un sándwich y un plátano, así como el agradecimiento.

Después, el laboratorio se encargó de brindar los resultados correspondientes. El estudio se apegó a lo dispuesto en el Reglamento de la Ley General de Salud en Materia de Investigación para la Salud ${ }^{17}$, así como lo establecido en la Declaración de Helsinki ${ }^{18}$. Posterior a la obtención de resultados, los datos fueron capturados en el paquete estadístico IBM SPSS versión 21.0, donde se realizó estadística descriptiva e inferencial.

\section{Resultados}

De acuerdo con los resultados, se pudo observar que el 59.0\% de los participantes fueron del sexo femenino, respecto al grado escolar, el $36.9 \%$ pertenecieron al sexto semestre, el $35.2 \%$ al cuarto semestre y por último el $27.9 \%$ al segundo semestre. La media de edad fue de $(\mathrm{M}=16.71, \mathrm{DE}=1.03)$, el peso promedio fue de $(\mathrm{M}=92.22, \mathrm{DE}=12.49)$, la talla de $(\mathrm{M}=162.21, \mathrm{DE}=22.52)$ y predominó la categoría de obesidad grado I (Tabla 1).

Tabla 1. Distribución según grados de obesidad ( $n=122)$

\begin{tabular}{lcc} 
Grado de Obesidad (IMC) & F & $\%$ \\
\hline Obesidad $^{\circ}$ I $(30.0-34.9)$ & 83 & 68.0 \\
Obesidad $^{\circ}$ II $(35.0-39.9)$ & 30 & 24.6 \\
Obesidad $^{\circ}$ III $(>40.0)$ & 9 & 7.4 \\
\hline
\end{tabular}

Se realizó un tabla de contingencia para verificar las agrupaciones entre los antecedentes familiares de enfermedades crónicas de los participantes y los grados de obesidad, en donde se encontró que las enfermedades crónicas, endocrinas y metabólicas de las madres, son los únicos antecedentes familiares que tienen una asociación con la obesidad de los adolescentes $\left(X^{2}=35.53, p=.001\right)$, lo cual puede indicar que son un factor predisponente para el desarrollo de obesidad a edad temprana (Tabla 2).

Tabla 2. Antecedentes familiares de enfermedades crónicas y grados de obesidad

\begin{tabular}{|c|c|c|c|c|c|c|}
\hline \multirow{3}{*}{$\begin{array}{l}\text { Antecedentes familiares de } \\
\text { enfermedades crónicas }\end{array}$} & \multicolumn{6}{|c|}{ Obesidad } \\
\hline & \multicolumn{2}{|c|}{$I(n=83)$} & \multicolumn{2}{|c|}{ II $(n=30)$} & \multicolumn{2}{|c|}{ III $(n=9)$} \\
\hline & $\mathbf{F}$ & $\%$ & $f$ & $\%$ & $f$ & $\%$ \\
\hline \multicolumn{7}{|l|}{ Abuelos Paternos } \\
\hline Sin antecedentes & 57 & 68.7 & 20 & 66.7 & 7 & 77.8 \\
\hline Con antecedentes & 26 & 31.3 & 10 & 33.3 & 2 & 22.2 \\
\hline \multicolumn{7}{|l|}{ Abuelos Maternos } \\
\hline Sin antecedentes & 56 & 67.5 & 18 & 60.0 & 4 & 44.4 \\
\hline Con antecedentes & 27 & 32.5 & 12 & 40.0 & 5 & 55.6 \\
\hline \multicolumn{7}{|l|}{ *Madre } \\
\hline Sin antecedentes & 62 & 74.7 & 16 & 53.3 & 2 & 22.2 \\
\hline Con antecedentes & 21 & 25.3 & 14 & 46.7 & 7 & 77.8 \\
\hline \multicolumn{7}{|l|}{ Padre } \\
\hline Sin antecedentes & 58 & 69.9 & 21 & 70.0 & 3 & 33.3 \\
\hline Con antecedentes & 25 & 30.1 & 9 & 30.0 & 6 & 66.7 \\
\hline \multicolumn{7}{|l|}{ Hermanos } \\
\hline Sin antecedentes & 75 & 90.4 & 27 & 90.0 & 7 & 77.8 \\
\hline Con antecedentes & 8 & 9.6 & 3 & 10.0 & 2 & 22.2 \\
\hline
\end{tabular}


En relación con los resultados de los criterios para el SM, se encontró que el criterio mayormente alterado fue la circunferencia de cintura, donde casi la totalidad de los participantes obtuvieron una medición mayor a $90 \mathrm{~cm}$. Seguido de la presión arterial donde el 41.8\% presentó percentil mayor a 90, lo que indica presión arterial elevada. Respecto a los resultados sanguíneos, el 97.5\% presentó niveles de glucosa inferiores a los $100 \mathrm{mg} / \mathrm{dL}$ que se traduce en niveles normales; en el colesterol HDL el 75.4\% tuvieron colesterol mayor a $40 \mathrm{mg} / \mathrm{dL}$ y en relación a los triglicéridos el 77.9\% obtuvo cifras menores de 110 $\mathrm{mg} / \mathrm{dl}$, lo que indica que en ambos casos más del 70\% resultaron clasificados con resultados normales.

En la tabla 3 se puede observar que algunos de los indicadores se ven alterados de acuerdo al grado de obesidad, particularmente los componentes de tipo sanguíneo: colesterol HDL y triglicéridos, tienden a presentarse en mayor proporción entre los grados de obesidad I y II respectivamente. Sin embargo, los componentes de la cintura y la presión arterial, se encuentran más alterados conforme el grado de obesidad es mayor.

De acuerdo con los componentes del fenotipo de Cook, para el desarrollo de SM, los resultados demuestran que el $25.4 \%$ de los participantes presentaron tres o más criterios alterados por lo que se consideran con SM. Al realizar un análisis de asociación entre los grados de obesidad y los criterios anteriores, se observó que únicamente los niveles de glucosa presentaron significancia estadística con los grados de obesidad $\left(X^{2}=9.43, p<.009\right)$, este resultado pudiera estar afectado por el número de casos, ya que en este indicador es donde hubo sólo tres casos con valores alterados (Tabla 3).

Tabla 3. Asociación entre criterios de síndrome metabólico con grados de obesidad

\begin{tabular}{|c|c|c|c|c|c|c|c|}
\hline \multirow{3}{*}{$\begin{array}{c}\text { Criterios de síndrome } \\
\text { metabólico }\end{array}$} & \multicolumn{7}{|c|}{ Obesidad } \\
\hline & & \multicolumn{2}{|c|}{$I(n=83)$} & \multicolumn{2}{|c|}{ II $(n=30)$} & \multicolumn{2}{|c|}{ III $(n=9)$} \\
\hline & & $\mathbf{F}$ & $\%$ & $f$ & $\%$ & $\mathbf{f}$ & $\%$ \\
\hline \multicolumn{8}{|l|}{ Circunferencia de Cintura } \\
\hline & $<90 \mathrm{~cm}$ & 1 & 1.2 & 1 & 3.3 & 0 & 0.0 \\
\hline & $\geq 90 \mathrm{~cm}$ & 82 & 98.8 & 29 & 96.7 & 9 & 100.0 \\
\hline \multicolumn{8}{|l|}{ Presión Arterial } \\
\hline & $<\mathrm{P} 90$ & 53 & 63.9 & 14 & 46.7 & 4 & 44.4 \\
\hline & $\geq \mathrm{P} 90$ & 30 & 36.1 & 16 & 53.3 & 5 & 55.6 \\
\hline \multicolumn{8}{|l|}{ *Glucosa } \\
\hline & $<100 \mathrm{mg} / \mathrm{dL}$ & 83 & 100.0 & 27 & 90.0 & 9 & 100.0 \\
\hline & $>100 \mathrm{mg} / \mathrm{dL}$ & 0 & 0.0 & 3 & 10.0 & 0 & 0.0 \\
\hline \multicolumn{8}{|l|}{ Colesterol HDL } \\
\hline & $<40 \mathrm{mg} / \mathrm{dL}$ & 25 & 30.1 & 4 & 13.3 & 1 & 11.1 \\
\hline & $>40 \mathrm{mg} / \mathrm{dL}$ & 58 & 69.9 & 26 & 86.7 & 8 & 88.9 \\
\hline \multicolumn{8}{|l|}{ Triglicéridos } \\
\hline & $<110 \mathrm{mg} / \mathrm{dL}$ & 67 & 80.7 & 21 & 70.0 & 7 & 77.8 \\
\hline & $>110 \mathrm{mg} / \mathrm{dL}$ & 16 & 19.3 & 9 & 30.0 & 2 & 22.2 \\
\hline
\end{tabular}

*Significancia estadística $(p<.01)$

\section{Discusión}

Para cumplir el objetivo de determinar los criterios para SM de mayor influencia en adolescentes con obesidad, se consideraron los criterios del NCEP-ATP III utilizado por Cook como criterios diagnósticos para el SM; conforme a estos una cuarta parte de los adolescentes con obesidad fueron clasificados con SM. Dichos resultados coinciden con los reportados por diversos autores ${ }^{7,19-21}$, quienes de igual manera obtuvieron una elevada prevalencia de SM en adolescentes con problemas de obesidad. Estos resultados tienden a ser coherentes, dado que como es conocido, la obesidad es un factor determinante para 
desarrollar dislipidemias y trastornos, tanto vasculares como endócrinos, independientemente de la edad de las personas.

No obstante, se ha encontrado literatura que difiere ${ }^{22-27}$, ya que se ha reportado una baja prevalencia del SM entre los adolescentes, a pesar de que presentaban obesidad. Por lo que se puede deducir que la adolescencia es una etapa en la que aún es posible revertir el desarrollo de SM, incluso cuando se presenten problemas de obesidad.

Así mismo, al igual que en el presente estudio, se puede observar en los resultados de otras investigaciones que la medición de la circunferencia abdominal es el criterio diagnóstico más frecuente ${ }^{19,26,27}$ y un buen predictor clínico del riesgo cardiovascular asociado a la obesidad abdominal, al igual que el IMC, ya que la acumulación de grasa en la zona abdominal se asocia también a un mayor riesgo de SM.

Por otro lado, en un estudio ${ }^{28}$ en el cual se reportó que el colesterol HDL reducido fue el componente más frecuente seguido de la hipertrigliceridemia, contrario en lo que respecta a lo encontrado en este estudio, el Colesterol HDL estuvo aumentado en mayor porcentaje y en el criterio de los triglicéridos, la mayor frecuencia fue menor a los $110 \mathrm{mg} / \mathrm{dl}$, por lo que se debe considerar que la alimentación es un factor determinante, para un mayor riesgo de enfermedades cardiovasculares y síndrome metabólico.

\section{Conclusiones}

La adolescencia es una etapa crucial para el diagnóstico oportuno de patologías desencadenadas por la obesidad, los hallazgos de ésta investigación demuestran una elevada prevalencia de SM entre los adolescentes con esta condición.

La obesidad abdominal es un claro signo de problemas relacionados con el peso y se presenta en casi la totalidad de los participantes. Es alarmante si se observa que una cuarta parte de la población joven con obesidad, a su corta edad han desarrollado un trastorno como lo es el SM, esto sin considerar los riesgos que conlleva como generar DM2, enfermedad arterial coronaria y cerebrovascular por arterioesclerosis, que son las principales causas de muerte en México.

También, se puede determinar que los antecedentes patológicos heredofamiliares maternos, juegan un papel importante en el desarrollo de la obesidad a temprana edad. Por lo que se considera indispensable realizar intervenciones desde una perspectiva interdisciplinar, tal como se llevó a cabo en este trabajo. Un enfoque interdisciplinar fomentaría hábitos saludables desde el hogar, y cambios en el estilo de vida que pueden ser impartidas por profesionales de la salud y la actividad física. Con los cuáles pueda ser posible disminuir el índice de obesidad en la población joven y por consiguiente el desarrollo de SM y sus futuras complicaciones.

De acuerdo a lo mencionado antes, la adolescencia es una etapa temprana para educar a las personas y fomentar una vida más sana que permita mantenerlos lejos del desarrollo de enfermedades crónico degenerativas, que actualmente representan un gran impacto negativo en los indicadores de salud de este país, mismo que ayudaría a reducir costos en el sistema sanitario y mejorar la calidad de vida de la futura población adulta.

\section{Responsabilidades Éticas}

Protección de personas y animales. Los autores declaran que en esta investigación no se han realizado experimentos en seres humanos ni en animales.

Confidencialidad. Los autores declaran que este trabajo no contiene ningún dato confidencial de los participantes.

Conflicto de intereses. Los autores declaran no tener conflicto de intereses.

Financiamiento. Para la realización de este trabajo se contó con financiamiento del Programa Integral de Fortalecimiento Institucional (PIFI).

Agradecimientos. Se agradece a los participantes del estudio, así como a la institución donde se permitió el acceso y sus dirigentes. 


\section{Referencias}

1. García-García E, De la Llata-Romero M, Kaufer-Horwitz M, et al. La obesidad y el síndrome metabólico. Un reto para los Institutos Nacionales de Salud. Rev. Invest. Clin. 2009; 61 (4): 337-46.

2. Organización Mundial de la Salud. Obesidad y Sobrepeso. Washington, D.C.: OMS; 2017. [Consultado 28 agosto 2017]. Disponible en: https://bit.ly/2woCAxV

3. Palma O, Shamah-Levy T, Franco A, et al. Encuesta Nacional de Salud y Nutrición 2006. Morelos: Instituto Nacional de Salud Pública 2006. [Consultado 27 agosto 2016]. Disponible en: https://bit.ly/2q8P7zn

4. Instituto Nacional de Salud Pública. Encuesta Nacional de Salud y Nutrición de Medio Camino 2016. Morelos: INSP; 2017.

5. Bridger T. Childhood obesity and cardiovascular disease. Paediatr Child Health. 2009; 14(3): 177-82.

6. American Diabetes Association. Diabetes y Sobrepeso. Arlington, VA: American Diabetes Association 2014. [Consultado 27 agosto 2017]. Disponible en: https://bit.ly/2kyblHF

7. Leal VS, De Lira PIC, Oliveira JS, et al. Excesso de peso em crianças e adolescents no Estado de Pernamburco, Brasil: prevalência e determinantes. Cad. saúde pública. 2012; 28(6): 1175-82. http://dx.doi.org/10.1590/S0102-311X2012000600016

8. Zimmet P, Alberti G, Kaufman F, et al. The metabolic syndrome in children and adolescents. Lancet 2007; 369(9579): 2059-61. https://doi.org/10.1016/S0140-6736(07)60958-1

9. Lizarzaburu-Robles JC. Síndrome metabólico: concepto y aplicación práctica. An. Fac. med. 2013; 74(4): 315-20. [Consultado 27 agosto 2017]. Disponible en: https://bit.ly/2qhy9ig

10. Chen W, Berenson GS. Metabolic syndrome: definition and prevalence in chlindren. J. pediatr. 2007; 83(1): 1-3. http://dx.doi.org/10.2223/JPED.1584

11. Albornoz-López R, Pérez-Rodrigo I. Nutrición y síndrome metabólico. Nutr. clín. diet. hosp. 2012; 32(3): $92-7$.

12. Cook S, Weitzman M, Auinger P, et al. Prevalence of Metabolic Syndrome Phenotype in Adolescents: Findings From the Third National Health and Nutrition Examination Survey 1988-1994. Arch Pediatr Adolesc Med. 2003; 157 (8): 821-7. https://doi.org/10.1001/archpedi.157.8.821

13. Romero-Velarde E, Campollo-Rivas O, Celis-de la Rosa A, et al. Factores de riesgo de dislipidemia en niños y adolescentes con obesidad. Salud pública Méx. 2007; 49(2): 103-8.

14. Villalpando S, Carrión C, Barqueras S, et al. Asociación entre índice de masa corporal, hiperglicemia y alteraciones de los componentes del síndrome metabólico en adolescentes mexicanos. Salud pública Méx. 2007; 49(supl.3):324-30.

15. Balas-Nakash M, Villanueva-Quintana A, Tawil-Dayan S, et al. Estudio Piloto para la identificación de indicadores antropométricos asociados a marcadores de riesgo de síndrome metabólico en escolares mexicanos. Bol. Med. Hosp. Infant. Mex. 2008; 65(2): 100-9.

16. Grove SK, Gray JR, Burns N. Investigación en enfermería. Desarrollo de la práctica enfermera basada en la evidencia. $6^{a}$ ed. España: Elsevier; 2016.

17. Secretaría de Salud. Reglamento de la Ley General de Salud en Materia de Investigación para la salud. Ciudad de México: SSA; 2014. [Consultado 11 abril 2016]. Disponible en: https://bit.ly/1SBpqPT

18. Asociación médica mundial. Declaración de Helsinki de la AMM. Principios éticos para las investigaciones médicas en seres humanos. Fortaleza: AMM; 2013. [Consultado 11 abril 2016]. Disponible en: https://bit.ly/2r2W2cs

19. Eyzaguirre F, Silva R, Román R, et al. Prevalencia de síndrome metabólico en niños y adolescentes que consultan por obesidad. Rev. Méd. Chile. 2011; 139(6): 732-8.

http://dx.doi.org/10.4067/S0034-98872011000600006

20. Özer S, Yilmaz R, Özlem-Kazanci N, et al. Higher HDL levels are a preventive factor for metabolic syndrome in obese Turkish children. Nutr. hosp. 2015; 31(1): 307-12.

https://doi.org/10.3305/nh.2015.31.1.7691 
21. Burrows R, Leiva L, Weistaub G, et al. Síndrome metabólico en niños y adolescentes: asociación con sensibilidad insulínica y con magnitud y distribución de la obesidad. Rev. Méd. Chile. 2007; 135(2): 174-81. http://dx.doi.org/10.4067/S0034-98872007000200005

22. Pajuelo J, Bernui I, Nolberto V, et al. Síndrome metabólico en adolescentes con sobrepeso y obesidad. An. Fac. Med. 2007; 68(2): 143-9. [Consultado 11 abril 2016]. Disponible en: https://bit.ly/2EzaTGJ

23. Cárdenas-Villareal VM, López-Alvarenga JC, Bastarrachea RA, et al. Prevalencia del síndrome metabólico y sus componentes en adolescentes de la ciudad de Monterrey, Nuevo León. Arch. cardiol. Méx. 2010; 80(1); 19-26. [Consultado 27 agosto 2017]. Disponible en: https://bit.ly/2q2B0vw

24. Cardoso-Saldaña GC, Yamamoto-Kimura L, Medina-Urrutia A, et al. Exceso de peso y síndrome metabólico en adolescentes de la Ciudad de México. Arch. cardiol. Méx. 2010; 80(1); 12-8. [Consultado 11 abril 2016]. Disponible en: https://bit.ly/2EQSjdg

25. Camarillo-Romero E, Domínguez-García MV, Amaya-Chávez A, et al. Dificultades en la clasificación del síndrome metabólico. El ejemplo de los adolescentes en México. Salud pública Méx. 2010; 52(6): 524-7. [Consultado 27 agosto 2017]. Disponible en: https://bit.ly/2yNzsuj

26. Guijarro-de Armas MG, Monereo-Megías S, Merino-Viveros M, et al. Prevalencia de síndrome metabólico en una población de niños y adolescentes con obesidad. Endocrinol. nutr. 2012; 59(3): 153-224. https://doi.org/10.1016/j.endonu.2012.01.003

27. Zaldívar-Ochoa JR, Domínguez-Redondo D. Niños y adolescentes obesos con síndrome metabólico. Medisan. 2012; 16(7): 1098-104. [Consultado 27 agosto 2017]. Disponible en: https://bit.ly/2Pw4kIY

28. Carvajal-Carvajal C. Niveles de lípidos sanguíneos en pacientes adultos del Cantón de Pococí, Costa Rica 2013. Rev Costarr Salud Pública. 2015; 23(2): 19-29. [Consultado: 11 abril 2016]. Disponible en: https://bit.ly/2qfjpvw 